\title{
Multiple giant clusters in percolation of random networks
}

\author{
Yang Zhang, Binghui Guo and Zhiming Zheng \\ LMIB and School of Mathematics and Systems Science \\ Beihang University \\ Beijing, China \\ zhangyang@ss.buaa.edu.cn
}

\begin{abstract}
Classical percolation processes on random networks always have only one giant cluster after the transition. Recently, percolation with multiple giant clusters has been observed and attracted much attention. In this paper, we show three simple but different models which would lead to multiple giant clusters. The evolution of these models is discussed respectively and they are supported by numerical simulations. The study of these new models may present a deep understanding of the emergence of multiple giant clusters in random networks.
\end{abstract}

Keywords-multiple giant clusters; percolation transition; random networks

\section{INTRODUCTION}

The theory of complex networks has enjoyed tremendous development in the last few decades [1], and percolation transition [2], which concerns the emergence of large-scale connectivity, has been one of the most widely studied subject on networks since its application in system robustness and information or epidemic spreading [3].

In general, a bond percolation process in networks can be described as follows, every time an unoccupied link is chosen to be occupied with some given rule, until all the bonds are occupied. A well studied model is Erdös-Rényi (ER) random network [4], in which all the unoccupied edges are chosen in uniform every time. Erdös and Rényi proved that at the critical point $t_{c}=1 / 2$, an unique giant cluster will emerge. These classical percolation transitions are observed in many other models, and there is always only one giant cluster existing. But this is not the case in many real-world processes, as multiple giant clusters may be observed in natural systems. A cluster aggregation model with freezing rate [5] and a recently studied explosive percolation model called BFW model [6] opened up the way to have multiple giant clusters, but their results rely on preventing the growing of giant clusters, either by freezing or by the high probability to add internal-cluster links. In our recent work [7], we studied the formation mechanism of multiple giant clusters and present a multi-ER model according to the analysis. Here, more simple model of percolation with multiple giant clusters will be introduced and studied.

\section{MULTI-ER MODEL}

Multi-ER model was introduced in our recent work [7], of which the detailed process is as follows.

\section{A. Model description}

Start from $N$ nodes with all links between them unoccupied, every round we choose an unoccupied link uniformly at random, denote the clusters connected by this link $i, j$, and their sizes are $s_{i}, s_{j}$ respectively. The link is then occupied at this time with a control probability

$$
p=\exp \left(-\alpha s_{i} s_{j} / N^{4 / 3}\right)
$$

otherwise we drop this link and go to the next round. Let $L$ be the total number of occupied links, we repeat the above steps to occupy links until a given time $t=L / N$ is reached. $\alpha \geq 0$ is a continuously tunable parameter which could control the number of giant clusters as we show later. If $\alpha=0$, $p=1$ for every chosen link, and the model reduces to ER network, if $\alpha>0, p$ is smaller for links between large clusters than those between small clusters, so large clusters is suppressed to be connected. The larger $\alpha$ is, the stronger that suppression effect is.

It is worth to note that such suppression is valid only if the size product $s_{i} s_{j}$ has at least the order $O\left(N^{4 / 3}\right)$, since for small clusters $p \approx 1$. According to the classical theory of ER model, the largest clusters at the critical point have the size of order $O\left(N^{2 / 3}\right)$ [8], so our model is designed to control the merging probability of the largest several clusters in the critical window, while the early evolution is almost the same as ER model.

\section{B. Simulation results and discussion}

To see the behavior of percolation in our model, we numerically implement the model. Given a specific parameter value $\alpha=0.8$, the sizes of the first three largest clusters (denoted by $C_{i}, i=1,2,3$ ) per node is measured as a function of time $t$. A typical realization of total scale $N=10^{6}$ is shown in Fig. 1, that two giant clusters $C_{1}, C_{2}$ appear at the same critical point $t_{c}=0.5$, their relative sizes $C_{1} / N, C_{2} / N$ grow respectively later as time goes on. Inset, we show the behavior in the critical region in detail, it is found that $C_{2}$ emerges a little later than $C_{1}$, which may be due to the finite size effect.

The underlying mechanism of multi-ER model to have multiple giant clusters is that the merging of the largest clusters is restricted, thus they can only grow respectively till giant clusters are formed. This is the key to have multiple giant clusters emerge in principle. The parameter $\alpha$ in the 
model reflects the control strength, obviously for larger $\alpha$, more clusters are restricted to connect, thus more giant clusters could coexist.

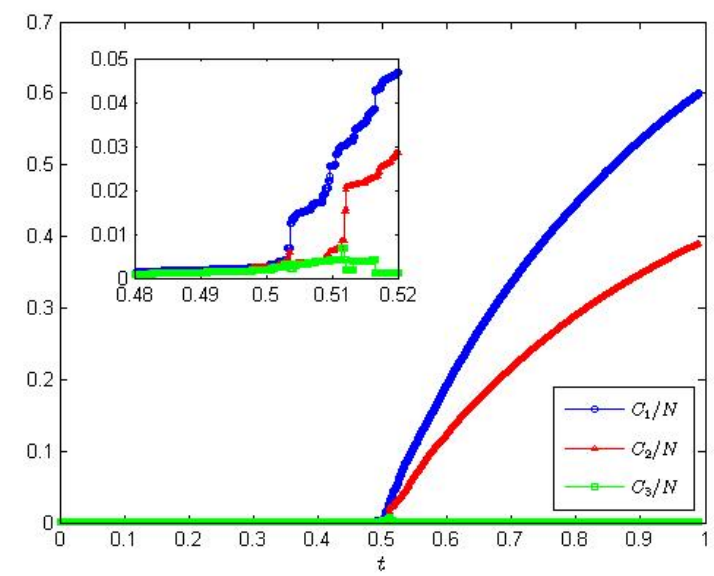

Figure 1. For $\alpha=0.8, C_{1} / N, C_{2} / N, C_{3} / N$ versus time $t$ in a typical realization, $N=10^{6}$. Inset is the behavior near the critical point $t_{c}=0.5$.

However, when we turn to the relation between the parameter $\alpha$ and the number of giant clusters, it is found that this is not deterministic. Explicitly speaking, for a given $\alpha$, the number of stable giant clusters has large fluctuations between different realizations. This phenomenon is mainly due to the randomness of the evolution in the critical window of this model.

We take $\alpha$ from 0 to 6 increased by 0.1 every time, for each $\alpha$ we numerically implement 1000 independent simulations, and record the number of stable giant clusters in every simulation. In practice, we stop occupying links at time $t=0.99$, and take giant clusters as the clusters of size larger than $0.01 N$. In all simulations, $N=10^{6}$. The results are shown in Fig. 2, in which the average, maximum and minimum number of giant clusters for every $\alpha$ are presented. We can see from the figure that the number of giant clusters increases with the parameter $\alpha$ as what we expected.

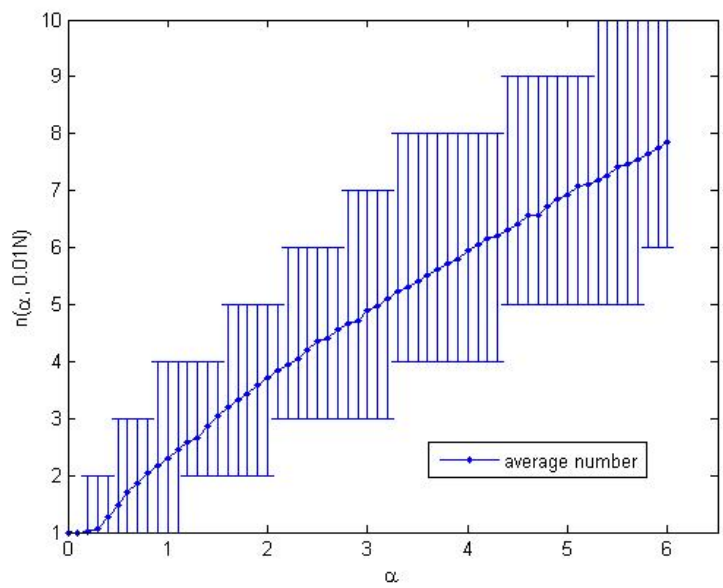

Figure 2. Number of stable giant clusters versus $\alpha, N=10^{6}$. The lower and upper bars are minimum and maximum values respectively.
We can have some variations of the model in which the number of giant clusters is deterministic. Note that if we replace the constant $N^{4 / 3}$ with $C_{i} C_{i+1}$ in (1) of the control probability $p$, then $i$ giant clusters emerge after the transition for sure, as any two of the first $i$ largest clusters have size product larger than $C_{i} C_{i+1}$, and thus are restricted to connect. This has been confirmed by numerical simulations.

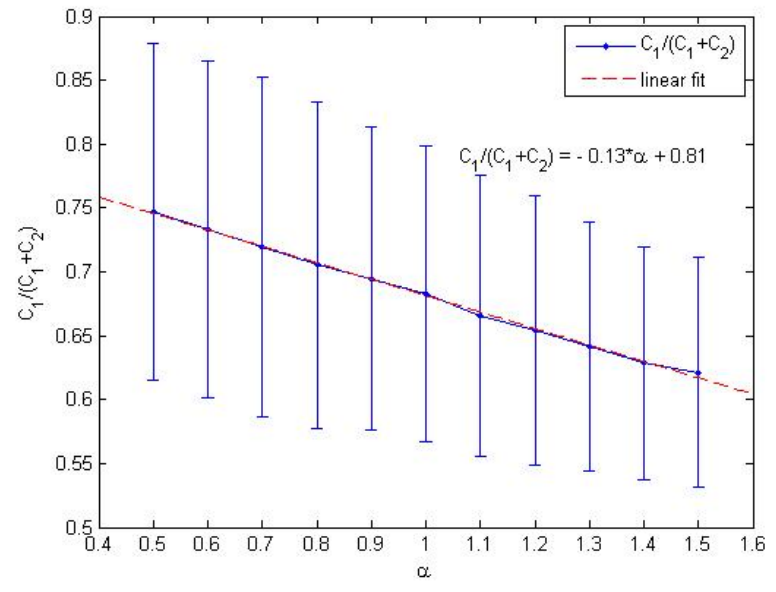

Figure 3. $C_{1} /\left(C_{1}+C_{2}\right)$ versus different $\alpha$.

Besides the number of giant clusters, the size ratio of different giant clusters is also studied, and shown in Fig. 3 for each $\alpha$. Large fluctuation also exists, but the average value has a linear relation with $\alpha$, the best linear fit is given.

\section{CONTROL THE GROWTH RATIO}

Inspired by the work of N. A. M. Araújo and H. J. Herrmann that control of the largest cluster could lead to explosive percolation [9], we discover a simple method to generate multiple giant clusters by control the growth ratio of the largest cluster at every step.

Every time we randomly choose a link just like the original ER model, denote $C_{1}, C_{1}{ }^{\prime}$ the size of the largest cluster before and after its occupation respectively, then if

$$
C_{1}^{\prime} \leq \max \left(r C_{1}, C_{1}+1\right)
$$

this link is accepted, otherwise we reject this link and return to choose another random one.

The parameter $r$ here is the maximum growth ratio allowed for the largest cluster in one step. Too large ratio of growth is forbidden. Obviously, $1 \leq r \leq 2$.

Different $r$ has large impact on the behavior of the process. In the limit, when $r=2$, no restriction exists as all other clusters are no larger than $C_{1}$. If $r<2$, the largest cluster is not allowed to connect with those clusters whose sizes are comparable with $C_{1}$. The smaller $r$ is, the more restrictions there are.

We can view the evolution of the model in Fig. 4 below, where $r$ is taken as 1.2 for every step, and the sizes of the first five largest clusters are shown versus $t$. The model has a transition near the critical point $t_{c} \approx 0.5$, and 4 giant clusters 
appear and grow since then. The simulation is done on a network of total vertices number $N=10^{6}$.

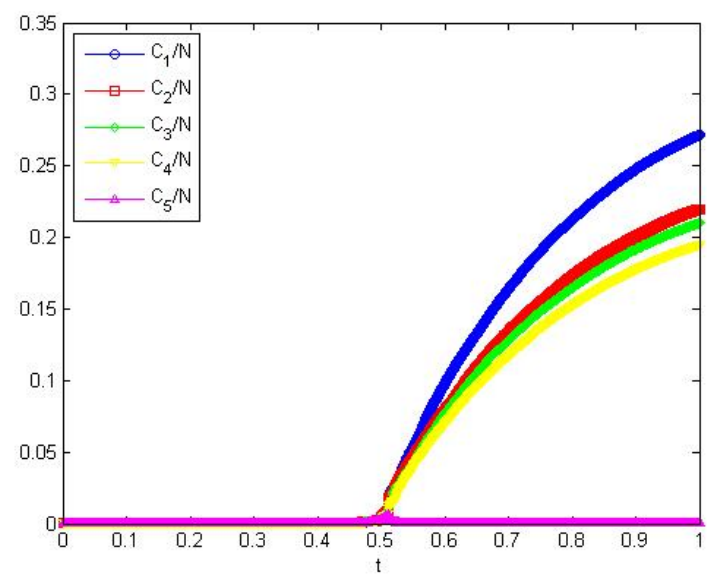

Figure 4. Size of the first five largest clusters versus $t(r=1.2)$

For different $r$, more simulation results show that when $r>1.5$, only one giant cluster can emerge, but it starts to have multiple giants for $r<1.5$, and as $r$ decrease, more giant clusters may appear. Surprisingly, $r$ seems to have no effect on the critical point, since $t_{c}$ always near 0.5 as we modify $r$. Note that 0.5 is just the critical point of ER model.

The model of control the growth ratio has many similarities with multi-ER model. Besides the same critical point, this model also has large fluctuation in the number and sizes of giant clusters. In percolation process, the largest several clusters must have similar sizes before the transition, if we control the growth ratio of the largest cluster, then the merging of these largest clusters is restricted. Thus the underlying mechanism for this model is consistent with multi-ER.

\section{FREEZING THE LARGEST CLUSTER}

The last model presented in this paper uses the idea similar to that in [5]. From the very beginning of the process, we find out the largest cluster, and denote it as $\mathbf{C}_{\mathbf{1}}$. In the evolution, we freeze $\mathbf{C}_{1}$ all the time, which means we do not choose any link that has one of its end nodes in $\mathbf{C}_{\mathbf{1}}$. Then after each step we should check if $\mathbf{C}_{\mathbf{1}}$ is replaced by a larger cluster through the link occupation. If so, we unfreeze the original one, and freeze the new $\mathbf{C}_{1}$ then.

In this process, $\mathbf{C}_{\mathbf{1}}$ is totally isolated to other clusters of the network, and the size of it can only increase when it is overtaken by the merging of two small clusters.

Simulations of this model are done. Unlike the above two models, two giant clusters will emerge for sure without fluctuation in their sizes. Furthermore, $\mathbf{C}_{\mathbf{1}}$ and $\mathbf{C}_{2}$ always have the same relative sizes after the transition.

In general, we can analytically learn the evolution of this model by comparing it to the classical ER model. Since the largest cluster is freezing, it is natural to view the network as two parts: cluster $\mathbf{C}_{\mathbf{1}}$ and the remaining networks.

We have two assumptions on this model:
1. The remaining network is approximate to an ER network, with $\mathbf{C}_{2}$ to be the giant cluster.

2. $\mathbf{C}_{1}$ and $\mathbf{C}_{2}$ must have almost the same quantities of the nodes and links in it.

With these two assumptions, we can calculate the sizes of the giant clusters versus time $t$ for this model.

Denote $C_{1}$ and $L_{1}$ the relative size of vertices and links in the largest cluster respectively. Then according to assumption 1, the remaining network is an ER network with a relative size $1-C_{1}$ of vertices and $t-L_{1}$ of links, so the relative time $t^{\prime}$ for the remaining network is

$$
t^{\prime}=\left(t-L_{1}\right) /\left(1-C_{1}\right) \text {. }
$$

For an ER network, the giant cluster size $\gamma$ and the time $t^{\prime}$ satisfy the equation as follows [10],

$$
1-\gamma=\exp \left(-2 t^{\prime} \gamma\right)
$$

According to the assumption 2 , the giant cluster in the remaining network has the same size with $\mathbf{C}_{1}$, thus

$$
\left(1-C_{1}\right) \gamma=C_{2}=C_{1} \text {. }
$$

To find out the number of links in the giant cluster of remaining network, it is worth to note that by the discrete duality principle [11], if we remove the giant cluster, it is still an ER network, with a relative size $t^{\prime}(1-\gamma)^{2}$ of links. Thus we can get $L_{1}$ as

$$
\left[t^{\prime}-t^{\prime}(1-\gamma)^{2}\right]\left(1-C_{1}\right)=L_{2}=L_{1} .
$$

Combing (3), (4), (5) and (6) together, we have

$$
\left(1-2 C_{1}\right) /\left(1-C_{1}\right)=\exp \left[-2 t C_{1} /\left(1-2 C_{1}^{2}\right)\right] .
$$

Solving the self-consistent equation (7), we can give an analytical fit of the giant cluster size in this model.

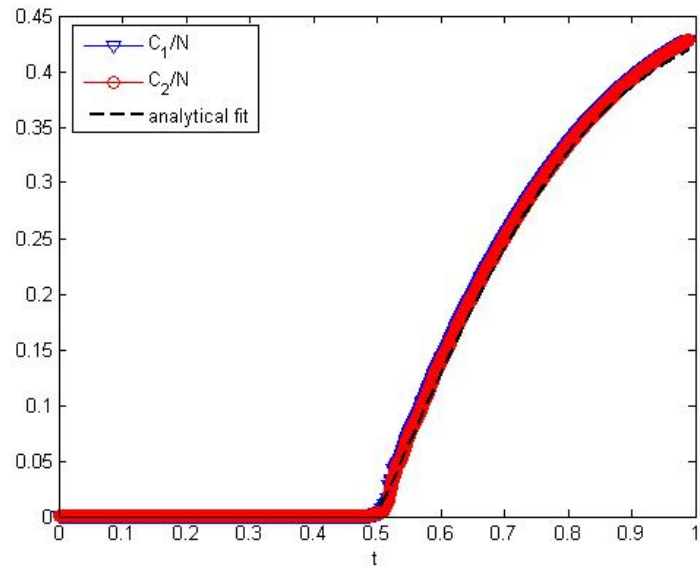

Figure 5. Evolution of $\mathbf{C}_{1}$ and $\mathbf{C}_{2}$ when freezing the largest cluster, dashed line is the analytical fit by solving (7). 
The sizes of the giant clusters versus $t$ for a realization of $N=10^{6}$ are shown in Fig. 5, note that $C_{1}$ and $C_{2}$ always have the same size as we described in the assumption, thus we can hardly distinguish them from each other in the figure. The dashed line is the analytical fit by (7), which coincides with the simulation very well and confirms our discussion.

This model can only have two giant clusters, for more ones, it is reasonable to consider of freezing more largest clusters.

\section{SUMMARY}

In this paper, we introduced three models of percolation with multiple giant clusters in random networks. These models are all based on simple modification of the classical ER model. They all exhibit a continuous transition at critical point $t_{c}=0.5$ just like the ER model. In the first two models, we can control the number of giant clusters by a parameter, but the number and sizes of giant clusters have large fluctuations. The third model is deterministic in that aspect, and we give an analytical calculation of it, but the number of giant clusters is not free to control.

Through these models the phenomenon of the emergence of multiple giant clusters in both theoretical and practical cases may be understood more deeply.

\section{ACKNOWLEDGMENT}

This work was supported by the Natural Science Foundation of China (Grant No. 11201019).

\section{REFERENCES}

[1] M. E. J. Newman, Networks: An introduction. New York: Oxford University Press, 2010

[2] D. Stauffer and A. Aharony, Introduction to Percolation Theory. London: Taylor \& Francis, 1994

[3] M. Sahimi, Applications of Percolation Theory. London: Taylor \& Francis, 1994.

[4] P. Erdös and A. Rényi, "On the evolution of random graphs" Publ. Math. Inst. Hung. Acad. Sci., vol. 5, 1960, pp. 17-61.

[5] E. Ben-Naim and P. L. Krapivsky, "Percolation with multiple giant clusters" J. Phys. A: Math. Gen., vol. 38, 2005, pp. L417-L423, doi:10.1088/0305-4470/38/23/L01.

[6] W. Chen and R. M. D'Souza, "Explosive Percolation with Multiple Giant Components" Phys. Rev. Lett., vol. 106, 2011, pp. 115701, doi: 10.1103/PhysRevLett.106.115701.

[7] Yang Zhang, Wei Wei, Binghui Guo, Renquan Zhang and Zhiming Zheng, "Formation mechanism and size features of multiple giant clusters in generic percolation processes" Phys. Rev. E, vol. 86, 2012, pp. 051103, doi:10.1103/PhysRevE.86.051103.

[8] B. Bollobás, Random Graphs, 2nd ed. Cambridge, England: Cambridge University Press, 2001.

[9] N. A. M. Araújo and H. J. Herrmann, "Explosive Percolation via Control of the Largest Cluster" Phys. Rev. Lett., vol. 105, 2010, pp. 035701, doi:10.1103/PhysRevLett.105.035701.

[10] M. E. J. Newman, S. H. Strogatz, and D. J. Watts, "Random graphs with arbitrary degree distributions and their applications" Phys. Rev. E, vol. 64, 2001, pp. 026118, doi: 10.1103/PhysRevE.64.026118.

[11] B. Bollobás, "The evolution of random graphs" Trans. Amer. Math. Soc., vol. 286, 1984, pp. 257-274, doi:10.1090/S0002-9947-19840756039-5. 